\title{
Turning yeast sequence into protein function
}

\section{Gunnar von Heijne}

By the beginning of April, the full DNA sequence of the Saccharomyces cerevisiae genome should have been completed. As reported at the recent "Perspectives in Protein Engineering" meeting (March 3-6, 1996, Montpellier, France), a mere $1 \%$ or so of the 12.5 million base yeast genome remained to be sequenced as of March 1. The question now is not "When will the data be available?" but "What will we do with it?".

Of the 6200-6300 potential open reading frames (ORFs) in the genome, around $25 \%$ have been sequenced and functionally characterized before: $30 \%$ encode proteins with significant similarity to proteins of known function from other organisms, and the remaining $40 \%$ represent completely novel proteins. Work is now underway (partly within the European Commission-funded EUROFAN project) to make a rapid assessment of the functions of these new genes by gene disruption, and, for some genes, by more detailed phenotypic studies.

The yeast data has already led to the identification of a number of proteins that had previously not been suspected to be part of the $S$. cerevisiae genetic repertoire. As André Goffeau (University of Louvain, Belgium) described in Montpellier, a large number of plasma membrane proteins with different transport functions have been identified by sifting through about half of the genome with hydrophobicity analysis and sequence alignment methods. Some of these are clearly homologous to the multidrug resistance proteins of bacteria and higher eukaryotes.

Gunnar von Heijne is at the department of biochemistry, Arrhenius Laboratory, Stockholm University, S-10691 Stockholm, Sweden

(gunnar@biokemi.su.se).

A particularly interesting example is PDR5, a protein that has all the hallmarks of the so-called ATP binding cassette $(\mathrm{ABC})$ proteins, but with the ATP-binding domains attached on the N-terminal rather than the Cterminal side of the membrane-embedded domains. As is usually the case for membrane

\section{IMAGE UNAVAILABLE FOR COPYRIGHT REASONS}

proteins, overproduction of this protein turned out to be difficult. However, a mutation in the PDR1 transcription factor was isolated that not only led to a 100 -fold increase in synthesis of active PDR5, but also allowed efficient sorting to the plasma membrane. Whether this or similar mutant strains will be a general panacea for membrane protein overproduction in yeast remains to be seen; the success with the PDR5/PDR1 system certainly suggests that genetic selection of overproducing strains may aid the assignment of protein function.

The rapid accumulation of fully sequenced prokaryotic and eukaryotic genomes puts new demands on bioinformatics. If, for instance, the sensitivity of sequence similarity searches can be increased by even a few percent, hundreds of new genes may be functionally and even structurally classified with minimal requirement for tedious experimental investigations. Thus, as Chris Sander of the European Bioinformatics Institute (EBI-EMBL, Cam- bridge, UK) pointed out, about $15 \%$ of all the yeast proteins are sufficiently similar to proteins of known 3D structure to allow automatic generation of $3 \mathrm{D}$ models. Such models are expected to be very accurate around the active site, fairly accurate within the central core of the protein, and unreliable in the exterior loop regions. They would be useful in, for instance, guiding site-directed mutagenesis studies.

Program packages such as GeneQuiz (http://www.embl-heidelberg.de/ casari/genequiz.html) or the software developed at the Institute for Genomic Research (Gaithersburg, MD) use a battery of sequence analysis routines to scan entire genomes. Results for 5000 yeast open ready frames (ORFs), as well as for the fully sequenced Haemophilus influenzae, Staphylococcus aureus, and Mycoplasma genitalium genomes are already out (Nature Biotechnology 14: 255-256, 1996) and more are coming.

Since analysis is dependent upon the quality of the databanks, the latest release of the widely used SWISS-PROT protein sequence database attempts to strike a new balance between quantity and quality, Rolf Apweiler of EBI-EMBL told the meeting. The SWISSPROT database is now supplemented with a new, automatically annotated database (TREMBL) including all ORFs from the EMBL nucleotide sequence database that are not already present in SWISS-PROT. Some 20,000 entries from TREMBL will eventually make it into SWISS-PROT, while other sequences will be transferred to specialized databases.

Between the large-scale sequencing projects and the World Wide Web, the long awaited unification of experimental and computational biology is finally happening. Where this will eventually take us is anybody's guess.

\section{In vivo veritas: Live phage display panning}

\section{Anna M. Wu}

Phage display of antibody fragments or peptides has provided a powerful method for generating molecules with novel binding characteristics $^{1}$. Bypassing immunization,

Anna $M$. Wu is an associate research scientist in the department of molecular biology, Beckman Research Institute of the City of Hope, 1450 East Duarte Road, Duarte, CA 91010 (awu@coh.org). libraries of filamentous phage displaying diverse antibody or peptide sequences are "panned" on purified antigen or whole cells ${ }^{2,3}$ in order to select for molecules that bind appropriately. Subsequent elution and amplification of the bound phage allows the recovery of proteins that can interact with targets previously inaccessible to conventional hybridoma technology; for instance, toxic substances or self-antigens ${ }^{4}$. Now, in the March 28 issue of Nature ${ }^{5}$, Pasqualini and
Ruoslahti from the La Jolla Cancer Research Foundation (La Jolla, CA), have extended phage display further, reporting successful "panning in vivo" to yield phage that home to specific organs.

It has been known for some time that lymphocyte and tumor cells can home to various organs by virtue of selective "address" molecules on endothelial surfaces 6 . As a more general approach to investigating the presence of such endothelial markers, 